\title{
Novel Technique for Closed Reduction of Incarcerated Medial Epicondyle from the Elbow Joint
}

\author{
Susanne H. Boden, $M D^{1}$; Stephanie A. Boden, $M D^{1}$; Z. Deniz Olgun, $M D^{2}$ \\ ${ }^{1}$ University of Pittsburgh Medical Center, Department of Orthopaedic Surgery, Pittsburgh, PA; ${ }^{2}$ Pediatric Orthopaedic \\ Division, UPMC Children's Hospital of Pittsburgh, University of Pittsburgh, Pittsburgh, PA \\ Correspondence to: Z. Deniz Olgun, MD; UPMC Children's Hospital of Pittsburgh, 4401 Penn Ave., Pittsburgh, PA 15224, \\ E-mail: olgunzd@upmc.edu
}

Received: December 29, 2021; Accepted: January 17, 2022; Published: February 1, 2022

DOI: 10.55275/JPOSNA-2022-0012

\begin{abstract}
:
While the majority of pediatric elbow dislocations are associated with a medial epicondyle fracture, an intra-articular incarcerated medial epicondyle is a rare sequela of closed elbow reduction. Elbow dislocations with incarceration of the medial epicondyle in the elbow joint have typically been considered an absolute indication for surgical intervention as closed manipulation is often unsuccessful according to the literature. We demonstrate a novel technique in which the incarcerated medial epicondyle can be extricated from the joint without having to go to the operating room, thus allowing for conservative management of these difficult injuries and reducing the risk of perioperative complications.

\section{Key Concepts:}

- The majority of pediatric elbow dislocations are associated with a medial epicondyle fracture.

- According to the literature, closed reduction techniques for intra-articular incarcerated medial epicondyle fractures are often unsuccessful.

- Incarceration of the medial epicondyle in the elbow joint is currently considered an absolute indication for surgery.

- We describe a technique for closed extraction of an incarcerated medial epicondyle fragment, which can be used in the ED setting to avoid urgent surgery and allow the consideration of non-operative treatment.
\end{abstract}

\section{Introduction}

Traumatic elbow dislocations are the most common type of dislocation in the pediatric patient population. They are associated with fractures of the medial epicondyle in up to $60 \%$ of cases. ${ }^{1-3}$ Posterolateral dislocations occur most frequently and have been shown to cause tension on the medial epicondyle via its attachment to the ulnar 
collateral ligament, the major stabilizing ligament of the elbow. ${ }^{1,4}$ The medial epicondyle ossification center usually does not fuse until late in the second decade of life, which is thought to place the medial epicondyle at increased risk of avulsion with pediatric elbow dislocations. ${ }^{3,5}$ Incarceration of the medial epicondyle in the elbow joint is a rare, but well-described sequela of closed elbow reduction. ${ }^{3-5}$ Intra-articular incarceration of the medial epicondyle has historically been considered an absolute indication for rapid open reduction, whereas elbow dislocations without medial epicondyle incarceration can either be managed nonoperatively or operated on semi-electively. ${ }^{3,6-8}$ We describe a new technique for closed extraction of incarcerated medial epicondyle using a simple maneuver, which may obviate the need for surgical intervention in these patients. The senior author has utilized this previously undescribed reduction maneuver in the operating room, and here we report an extended application of this maneuver in the ED setting.

\section{Description of the Method}

\section{Patient Presentation}

A 13-year-old Caucasian male presented to our Children's Emergency Department (ED) one day after a ground level fall while throwing a football sustaining a right elbow dislocation with an associated medial epicondyle fracture (Figure 1). The patient had initially presented to an outside facility, where he underwent closed reduction of his right elbow dislocation under conscious sedation. Post-reduction $\mathrm{x}$-rays obtained at that time demonstrated an incarcerated medial epicondyle in his joint (Figure 2a) for which he was referred to our $E D$, and orthopaedic surgery was consulted for further management.

\section{Clinical Evaluation and Reduction Technique}

Physical examination at the time of presentation to our ED demonstrated neurovascular integrity. After lengthy discussion of risks/benefits of management options, it was decided to attempt closed extraction of the incarcerated medial epicondyle in the ED without sedation using the reduction technique that the senior author utilizes in the operating room. With the forearm in supination, the elbow flexed between 45 and 60 degrees, and the wrist and fingers extended, the elbow was gently shaken back and forth in a varus-valgus direction (Figure 3). Repeat x-rays were obtained after this maneuver and demonstrated successful extraction of the intra-articular medial epicondyle (Figure 2b). The patient was placed in a long arm cast and discharged from the ED.

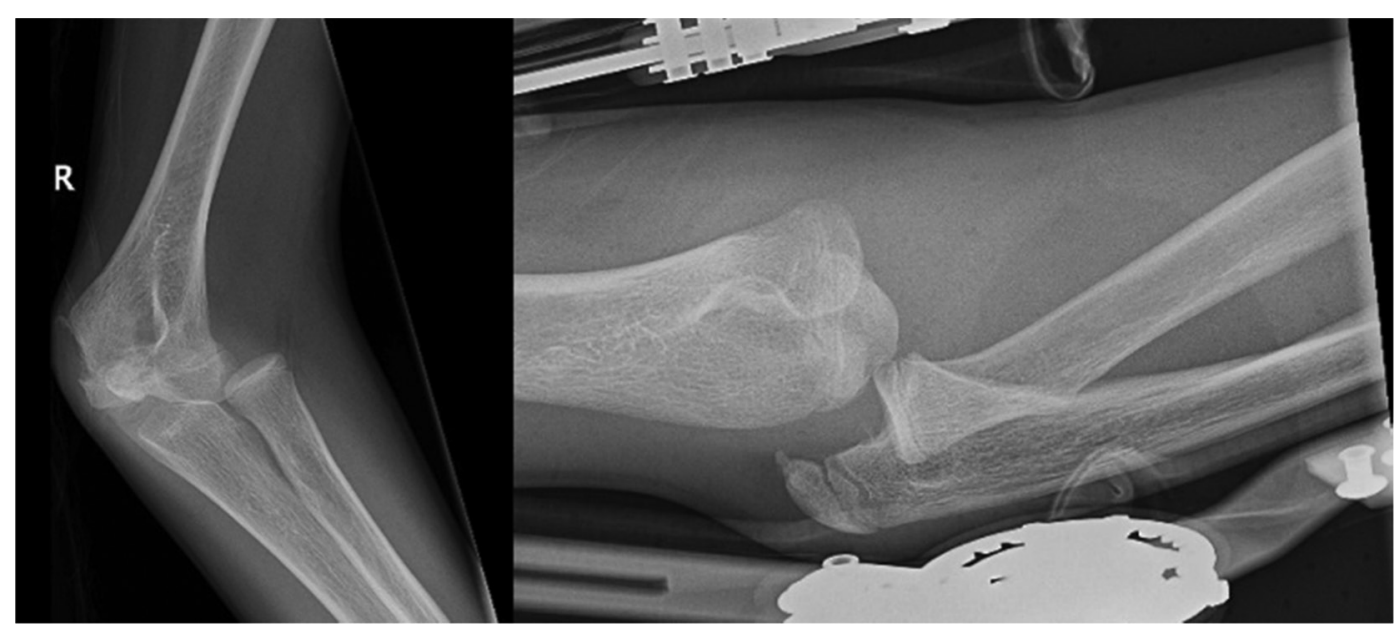

Figure 1. Radiographs taken before attempted reduction demonstrating a posterolateral elbow dislocation with an associated medial epicondyle fracture. 

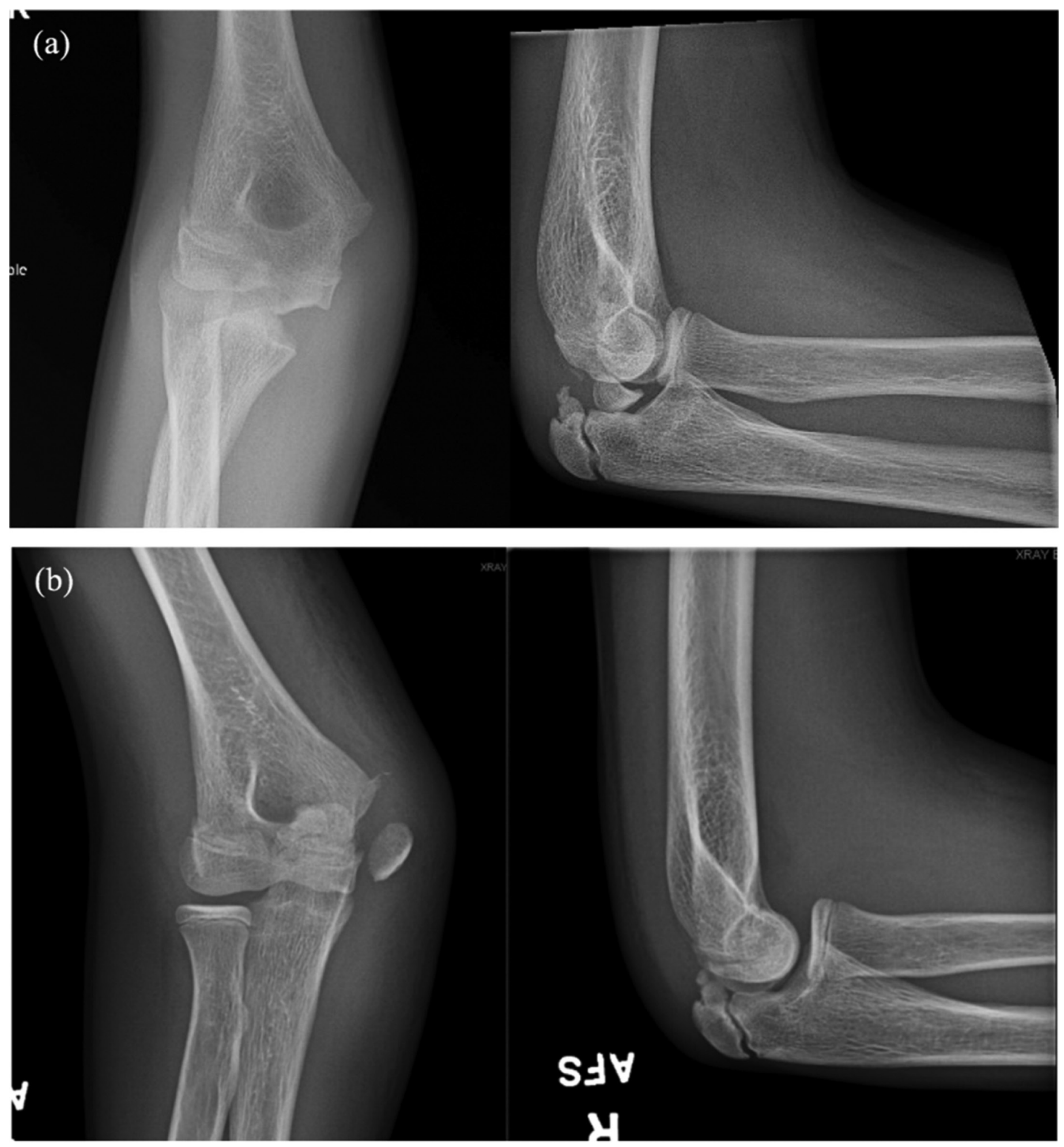

Figure 2. (a) Radiographs taken after initial attempt at closed reduction, demonstrating medial epicondyle fracture with intra-articular incarceration. (b) Radiographs taken after closed reduction via novel maneuver demonstrating extraction of incarcerated medial epicondyle with maintenance of elbow reduction. 

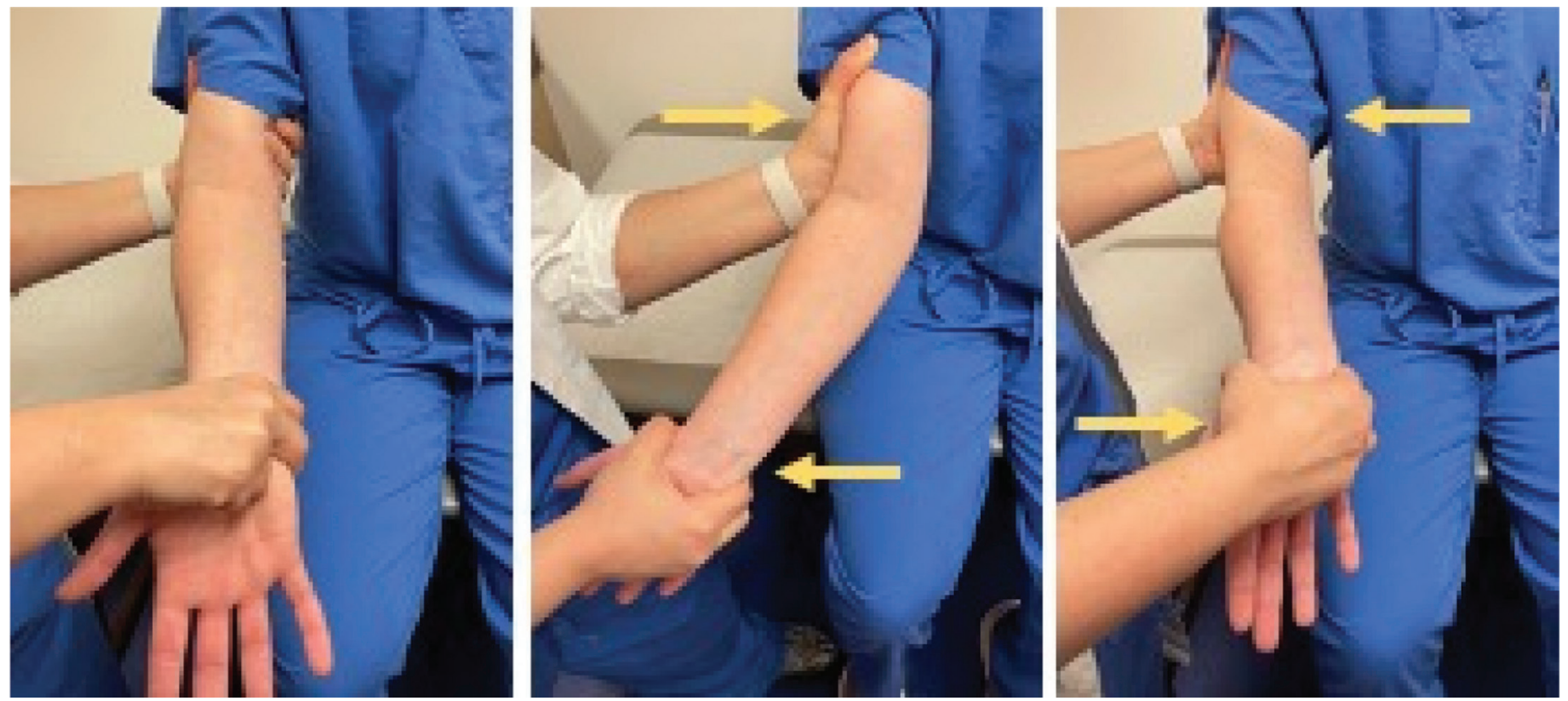

Figure 3. With the forearm supinated, wrist and fingers in extension, and elbow flexed around 45 degrees, the elbow is gently shaken back and forth in a varus-valgus direction. The yellow arrows represent the direction of force applied during this maneuver.

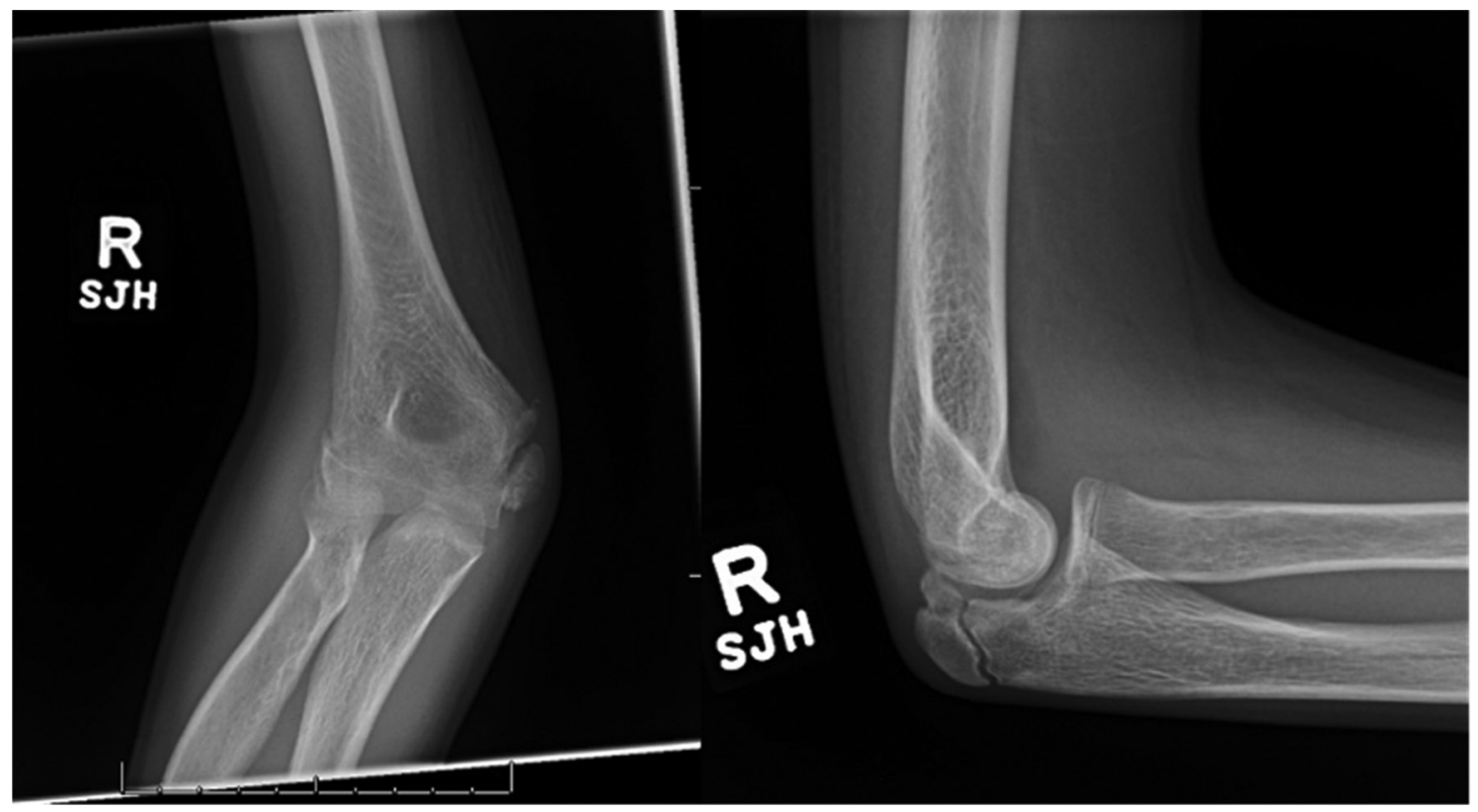

Figure 4. AP and lateral radiographs taken at 3-week follow-up after cast removal.

\section{Post-reduction Care}

At his follow-up appointment, repeat x-rays demonstrated acceptable alignment of the medial epicondyle fracture (Figure 4). The patient was treated conservatively with a long arm cast for 3 weeks and then transitioned out of his cast with initiation of early passive range of motion. On follow-up he had no ulnar nerve symptoms, was able to extend 5 degrees shy of full extension, and had stable positioning of the medial epicondyle fracture. 


\section{Comparison to Other Methods}

Intra-articular incarceration of the medial epicondyle following closed reduction of an elbow dislocation is a rare but potentially serious complication, which has traditionally constituted an absolute indication for surgical treatment via an open approach, extrication of the medial epicondyle, reduction and internal fixation thereof. ${ }^{1-3,9}$ Various techniques for reduction of an incarcerated fragment have been described in the literature, including the Esmarch technique and the Roberts maneuver. ${ }^{3,10,11}$

In the Esmarch technique, which is described as a method of open reduction in the operating room, an elastic bandage is used to compress the soft tissues from distal to proximal toward the fracture site to assist in reduction. ${ }^{3,11}$ In the Roberts maneuver, which was first described in 1934, the forearm is supinated with the fingers and wrist extended while a valgus stress is placed on the elbow and the elbow is repeatedly flexed and extended. . $^{3,10}$ This maneuver attempts to use the muscle forces of the attached common flexor muscles to extricate the entrapped fragment from the joint. Similarly, Dorman et al., described the use of a nerve stimulator to cause contraction of the flexor muscles, thereby indirectly reducing the incarcerated medial epicondyle. ${ }^{12}$ Despite these described techniques, closed manipulation is often unsuccessful according to the literature, and forceful manipulation causes concern for articular cartilage damage and potential nerve injury. ${ }^{9}, 12$ Additionally, there are currently no longterm follow-up studies or large case series reporting either success rates or clinical outcomes of the various closed reduction methods that have been historically described.

Open reduction and internal fixation (ORIF) for incarcerated medial epicondyle fractures is welldescribed in the literature with satisfactory outcomes. ${ }^{9}$ However, ORIF is not a benign procedure, and postoperative complications have been reported to occur at rates up to $31 \% .^{1,2}$ Our technique for closed reduction of an incarcerated medial epicondyle adds a new maneuver to the limited arsenal of previously described approaches. We demonstrate that with gentle shaking of the flexed elbow, the incarcerated medial epicondyle can extricate from the joint. This maneuver can be performed at the time of elbow reduction if recognized or later after post-reduction radiographs reveal an incarcerated medial epicondyle. When successful, this simple maneuver obviates the absolute surgical indication and can allow conservative management of these difficult injuries, potentially reducing the need for surgical treatment and therefore diminishing the risk of complication. Further studies should evaluate the success rate of this maneuver as well as longer-term clinical outcomes after closed reduction using this technique.

\section{Summary}

Intra-articular incarceration of the medial epicondyle has traditionally been considered an absolute indication for surgical intervention. Our varus-valgus reduction maneuver can successfully extricate the incarcerated medial epicondyle fragment without the use of anesthesia, therefore potentially eliminating the need for surgical management. While anecdotally this maneuver has been successful numerous times in the operating room over the years, in this report we demonstrate that the maneuver can work in the nonoperative setting. We do not recommend this method if the patient has concurrent nerve deficit; in this setting, open reduction with nerve exploration may be safer. In conclusion, this novel technique for closed reduction of an incarcerated medial epicondyle fractures provides a simple method of closed fragment extraction that can be used in an attempt to avoid open reduction and the potential for related complications.

\section{Additional Links}

- Outcomes of Nonoperative Pediatric Medial Humeral Epicondyle Fractures With and Without Associated Elbow Dislocation: Isolated medial epicondyle fractures with or without elbow dislocation can successfully be treated nonoperatively in the absence of absolute surgical indications. ${ }^{8}$ https://pubmed.ncbi.nlm.nih.gov/27741036/

- Medial Epicondyle Fractures in the Pediatric Population: An incarcerated intra-articular medial epicondyle is an absolute indication for surgical intervention. ${ }^{3} \mathrm{https}$ :// pubmed.ncbi.nlm.nih.gov/22474092/ 


\section{References}

1. Dodds SD, Flanagin BA, Bohl DD, et al. Incarcerated medial epicondyle fracture following pediatric elbow dislocation: 11 cases. J Hand Surg Am. Sep 2014;39(9):1739-1745. doi: https://doi.org/10.1016/j. jhsa.2014.06.012.

2. Tarallo L, Mugnai R, Fiacchi F, et al. Pediatric medial epicondyle fractures with intra-articular elbow incarceration. J Orthop Traumatol. Jun 2015;16(2):117-123. doi: https://doi.org/10.1007/s10195-014-0310-2.

3. Gottschalk HP, Eisner E, Hosalkar HS. Medial epicondyle fractures in the pediatric population. J Am Acad Orthop Surg. Apr 2012;20(4):223-232. doi: https://doi.org/10.5435/JAAOS-20-04-223.

4. Kaziz H, Naouar N, Osman W, et al. Outcomes of paediatric elbow dislocations. Malays Orthop J. Mar 2016;10(1):44-49. doi: https://doi. org/10.5704/MOJ.1603.008.

5. Chessare JW, Rogers LF, White H, et al. Injuries of the medial epicondylar ossification center of the humerus. AJR Am J Roentgenol. Jul 1977;129(1):49-55. doi: https://doi.org/10.2214/ajr.129.1.49.

6. Fowles JV, Slimane N, Kassab MT. Elbow dislocation with avulsion of the medial humeral epicondyle. J Bone Joint Surg Br. Jan 1990;72(1): 102-104. doi: https://doi.org/10.1302/0301-620X.72B1.2298765.
7. Patrick J. Fracture of the medial epicondyle with displacement into the elbow-joint. J Bone Joint Surg Am. Jan 1946;28:143-147.

8. Knapik DM, Fausett CL, Gilmore A, et al. Outcomes of nonoperative pediatric medial humeral epicondyle fractures with and without associated elbow dislocation. J Pediatr Orthop. Jun 2017;37(4):e224-e228. doi: https://doi.org/10.1097/BPO.0000000000000890.

9. Vuillermin C, Donohue KS, Miller P, et al. Incarcerated Medial Epicondyle Fractures With Elbow Dislocation: Risk Factors Associated With Morbidity. J Pediatr Orthop. Oct 2019;39(9):e647-e651. doi: https:// doi.org/10.1097/BPO.0000000000000991.

10. Roberts NW. Displacement of the internal epicondyle into the elbow-joint: four cases successfully treated by manipulation. Lancet. 1934;2:78-79.

11. Kamath AF, Cody SR, Hosalkar HS. Open reduction of medial epicondyle fractures: operative tips for technical ease. J Child Orthop. Aug 2009;3(4):331-336. doi: https://doi.org/10.1007/s11832-009-0185-6.

12. Dorman S, Sripada S, Clift BA, et al. Extraction of incarcerated medial epicondyle from the elbow joint using conventional nerve stimulator: a case report. J Med Case Rep. Oct 07 2014;8:329. doi: https://doi. org/10.1186/1752-1947-8-329. 